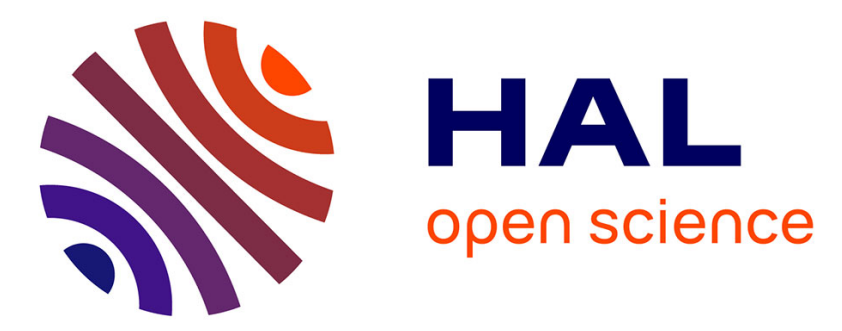

\title{
A computational approach for the lifetime prediction of cardiovascular balloon-expandable stents
}

Ferdinando Auricchio, Andrei Constantinescu, Michele Conti, Giulia Scalet

\section{To cite this version:}

Ferdinando Auricchio, Andrei Constantinescu, Michele Conti, Giulia Scalet. A computational approach for the lifetime prediction of cardiovascular balloon-expandable stents. International Journal of Fatigue, 2015, 75, pp.69-79. 10.1016/j.ijfatigue.2015.02.002 . hal-01219744

HAL Id: hal-01219744

https://hal-polytechnique.archives-ouvertes.fr/hal-01219744

Submitted on 6 May 2020

HAL is a multi-disciplinary open access archive for the deposit and dissemination of scientific research documents, whether they are published or not. The documents may come from teaching and research institutions in France or abroad, or from public or private research centers.
L'archive ouverte pluridisciplinaire HAL, est destinée au dépôt et à la diffusion de documents scientifiques de niveau recherche, publiés ou non, émanant des établissements d'enseignement et de recherche français ou étrangers, des laboratoires publics ou privés.

\section{(c)(1)}

Distributed under a Creative Commons Attribution| 4.0 International License 


\title{
A computational approach for the lifetime prediction of cardiovascular balloon-expandable stents
}

\author{
F. Auricchio ${ }^{\mathrm{a}}$, A. Constantinescu ${ }^{\mathrm{b}}, \mathrm{M}$. Conti ${ }^{\mathrm{a}}$, G. Scalet ${ }^{\mathrm{a}, *}$ \\ a Dipartimento di Ingegneria Civile e Architettura, Università di Pavia, Via Ferrata 3, 27100 Pavia, Italy \\ ${ }^{\mathrm{b}}$ Laboratoire de Mécanique des Solides - CNRS UMR 7649, École Polytechnique, 91128 Palaiseau cedex, France
}

\begin{abstract}
This paper presents a methodology for the numerical fatigue-life assessment of cardiovascular balloon-expandable stents. The methodology is based on a global computational approach composed of a mechanical finite element analysis, followed by a fatigue analysis. The method is applied to a classical $316 \mathrm{~L}$ stainless steel coronary stent design (i.e., the Medinol/Boston Scientific NIR ${ }^{\mathrm{TM}}$ stent). Fatigue criteria based on elastic and plastic shakedown concepts for finite and infinite lifetime are used to predict fatigue crack initiation and are calibrated on experimental data related to $316 \mathrm{~L}$ stainless steel $\mu \mathrm{m}$-size compo-nents, manufactured as stents. The results from the fatigue analysis allow to discuss several aspects affecting stent lifetime, such as the applied cyclic loading including systolic-diastolic pressurization and bending. The generality of the proposed methodology encourages further investigations of such an approach for its application to other materials or small-scale components.
\end{abstract}

\section{Introduction}

Nowadays, cardiovascular diseases represent a global health care problem, since are the leading cause of death and illness accounting for $30 \%$ of the deaths worldwide annually [1]. The advantage of stenting intervention with respect to the classical bypass surgery has been largely demonstrated in the treatment of heart disease [2] and now stents are involved in more than $85 \%$ of coronary intervention procedures [1]. Stents are small tube-like devices used to restore patency of blood vessels having the lumen area reduced due to atherosclerosis. The stent acts as a mechanical scaffold for the vessel and its implant is performed by a minimally invasive procedure inserting a catheter through a small incision in the femoral artery.

Several materials, as the $316 \mathrm{~L}$ austenitic stainless steel, the cobalt-chromium alloy or the NiTi-based shape memory alloy, are well-known in the biomedical field [3-6] and one of their biomedical applications regards coronary stents [7]. As an example, the 316L stainless steel is used for the PalmazSchatz (Cordis) or the Medinol/Boston Scientific NIR ${ }^{\mathrm{TM}}$ balloon-expandable stents, thanks to its good properties in the fully annealed condition, e.g., well adapted mechanical characteristics (i.e., great ductility, high

\footnotetext{
* Corresponding author. Tel.: +390382 985468.

E-mail address: giulia.scalet@unipv.it (G. Scalet).
}

tensile strength, and a raised elastic limit), biocompatibility, resistance to corrosion, as well as fatigue performances [5].

Although stent design is a relatively mature topic, fatigue failure has recently appeared as one of the main cause of stent drawback [1,8-12]. In particular, stents should survive at least for 10 years without exhibiting failure, that correspond to $4 \cdot 10^{8}$ systolic-diastolic pulsatile cycles [13]. Therefore, stent design lies within the high- and very high-cycle fatigue regime (denoted, respectively, as HCF and VHCF) [14]. Several parameters significantly affect the fatigue performance of stents and increase the complexity of the design process; among the others, we can cite the loading during the implant, the small-size of the device, manufacturing-induced features, complex material behavior, corrosive environment [1]. Stents have to withstand both their initial deployment within the artery, which involves large amounts of plastic strains, and the fatigue loading due to blood pressure, which can cause the failure of the stent even at stress levels lower than the tensile or yield strength of the material $[15,16]$. The majority of stents are manufactured by drawing a very fine tube, followed by laser cutting, leading to a final structure composed of struts connected by hinges [17]. Current stent strut cross sections are approximately $50-150 \mu \mathrm{m}$, which allow only for a few grains across (approximately 10-20), thus affecting fatigue resistance and making the application of standard bulk models inadequate [17-20]. The manufacturing process induces also the presence of stress concentration features, in particular, of small radius connections affecting fatigue behavior [21]. 
Therefore, a deep understanding of the fatigue and fracture failure in microsize components is necessary, in order to increase stent durability. Despite the importance of such applications and the associated difficulty, most of the research work on stent failure is based on medical statistics and/or clinical studies [1] and the literature disposes of few experimental data sets; see, e.g., [21-27] for $316 \mathrm{~L}$ stainless steel stents. Most of them refer to $10^{7}$ cycles, equivalent to an implant period of about 3.2-4 months due to systolic-diastolic pulsatile loading.

Several computational tools, as finite element (FE) analysis, have already proved their usefulness to bridge the gap between the existing experiments and the design of such components, e.g., [28-34]. Among the others, some works deal with the numerical fatigue-life assessment of metallic stents, e.g., [17,35-41]; most of them predict lifetimes lower than the required $4 \cdot 10^{8}$ cycles and do not focus on data from real precise experiments on stent components. Sweeney et al. [39] presented a refined FE stent fatigue methodology based on crystal plasticity theory for the prediction of crack initiation, using microstructural fatigue indicator parameters. The proposed simple criteria are however calibrated on experiments related to macroscopic specimens [42]. On the contrary, Sweeney et al. [43] presented the development of a micromechanical framework for stent fatigue design, based on a suitable experimental characterization of a biomedical $\mathrm{CoCr}$ alloy. Additionally, many computational modeling works on stents directly apply bulk material properties to the structure [30,36,37,40,44,35,41]. Among these works, dos Santos et al. [36] predicted lifetimes lower than $4 \cdot 10^{8}$ cycles for balloon-expandable stainless steel stents by incorporating a two-scale plasticity-damage model [45]. Such an approach, however, requires the calibration of several parameters, which has been done by referring to experimental data on macroscopic specimens. Barrera et al. [37] applied the multiaxial HCF criterion by Dang Van [46] to cardiovascular stents. Such a criterion, based on a micro-macro approach, successfully meets the need of microstructural representation in crack initiation predictions. However, the work by Barrera et al. [37] does not investigate the experimental data used for the criterion calibration [22,21] and constructs the Dang Van line, referring to only $10^{6}$ cycles, by assuming the same fatigue limits in bending and torsion. The work by Azaouzi et al. [40] predicts the fatigue-life of 316L stainless steel stents through the well-known Goodman diagram and the theory of critical distance based on the results presented in [22,21]. The Goodman parameters are again calibrated on experimental data $[22,21]$ without a detailed investigation.

The aim of the present paper is the introduction of a global computational design method for the lifetime assessment of balloonexpandable stents. The proposed prediction methodology is composed of a three-dimensional mechanical analysis, followed by a fatigue analysis. The mechanical analysis calculates the elastic or plastic shakedown mechanical state of the stent under investigation, by assuming the structure, loading, and material behavior known. The fatigue analysis is then based on the investigation of the stabilized state obtained within the mechanical analysis and evaluates the number of cycles to failure. Failure is here considered as a crack initiation, since the crack propagation phase is small with respect to the global time and the involved material thicknesses are rather small.

The stent geometry discussed here is a classical coronary stent design, i.e., the Medinol/Boston Scientific NIR ${ }^{\mathrm{TM}}$ stent, made of $316 \mathrm{~L}$ stainless steel. Due to practical difficulties in testing $\mu \mathrm{m}$-size specimens up to $4 \cdot 10^{8}$ cycles, we consider fatigue criteria calibrated on experimental data taken from the literature and related to smooth and notched $316 \mathrm{~L}$ steel $\mu \mathrm{m}$-size components used during industrial stent manufacturing $[21,22,27]$, starting from the work by
Auricchio et al. [47]. Particularly, in case of elastic shakedown, we focus on the multiaxial HCF criterion by Dang Van [46] and, in case of plastic shakedown, on the dissipated energy per cycle criterion [48,49]. Since the Dang Van criterion in [47] refers to a fatigue life of $10^{7}$ cycles, the present work proposes also an extension of such a calibration in order to investigate the behavior of the stent at the required $4 \cdot 10^{8}$ cycles [13].

The results from the mechanical and fatigue analysis are discussed with the purpose of providing a general discussion on the increased or decreased stent lifetime due to several conditions affecting the cardiovascular implant. Since coronary stent fatigue lifetime is affected by other cyclic movements in addition to the diastolic-systolic physiological loading (e.g., vessel deformations during the cardiac contraction [50,51]), we also explore the effect of bending cyclic loading associated with vessel curvature. The complexity of the analysis, modeling assumptions, as well as the difficulties that can be encountered are highlighted.

The proposed approach is general and applicable to several metallic materials as $316 \mathrm{~L}$ stainless steel or cobalt-chromium alloys, or to other micro-scale components.

The present paper is organized as follows. Section 2 presents the mechanical analysis of the stent. Then, Section 3 describes the results of the fatigue analysis. Conclusions and perspectives are finally given in Section 4.

\section{Mechanical analysis}

In this Section we illustrate the mechanical analysis performed with the FE method to compute the loading and the stabilized state of the stent during and after the implant.

\subsection{Stent: geometrical modeling and mesh}

We adopt a stent model resembling the design of the Medinol/ Boston Scientific NIR ${ }^{\mathrm{TM}}$ stent, which is nowadays a classical coronary stent design.

The geometry of the adopted stent model is based on the information reported by Sweeney et al. [39]. The generation of such a model is defined by a series of steps, detailed in the following. We firstly create a planar computer aided design representation of the stent, using the software Rhinoceros v.4.0 (McNeel \& associates, Seattle, WA, USA). The unfolded geometry of the device can be constructed by mirroring and replicating a primitive geometry (see Fig. 1a). The FE mesh is obtained by meshing the primitive geometry (see Fig. 1b) and similarly by mirroring and replicating it, as shown in Fig. 1c. The software used for this operation is Abaqus v.6.11 (Simulia, Dassault Systems, Providence, RI, USA). The final mesh of the stent is presented in Fig. $1 \mathrm{~d}$.

The whole stent model has an outer diameter of $0.876 \mathrm{~mm}$ and a length of $8.049 \mathrm{~mm}$. As depicted in Fig. 1a, both strut thickness and width are approximately $0.10 \mathrm{~mm}$. We remark that the characteristic dimensions of the stent geometry are comparable to the dimensions (ranging from 0.05 to $0.15 \mathrm{~mm}$ ) of the specimens $[22,21,27]$, on which the fatigue criteria are calibrated. The mesh is defined by 89,760 nodes and 57,600 three-dimensional brick elements (C3D8) available in the element library of the software Abaqus v.6.11, used as FEA solver. Taking into account the grain size given in [22,21,27], the actual mesh has about 2 elements per grain. To estimate possible errors due to large spatial gradients, we also adopt quadratic brick elements (C3D20R) for the same stent mesh. We can report that it causes an important increase of computational time without a noticeable improvement of the results. 

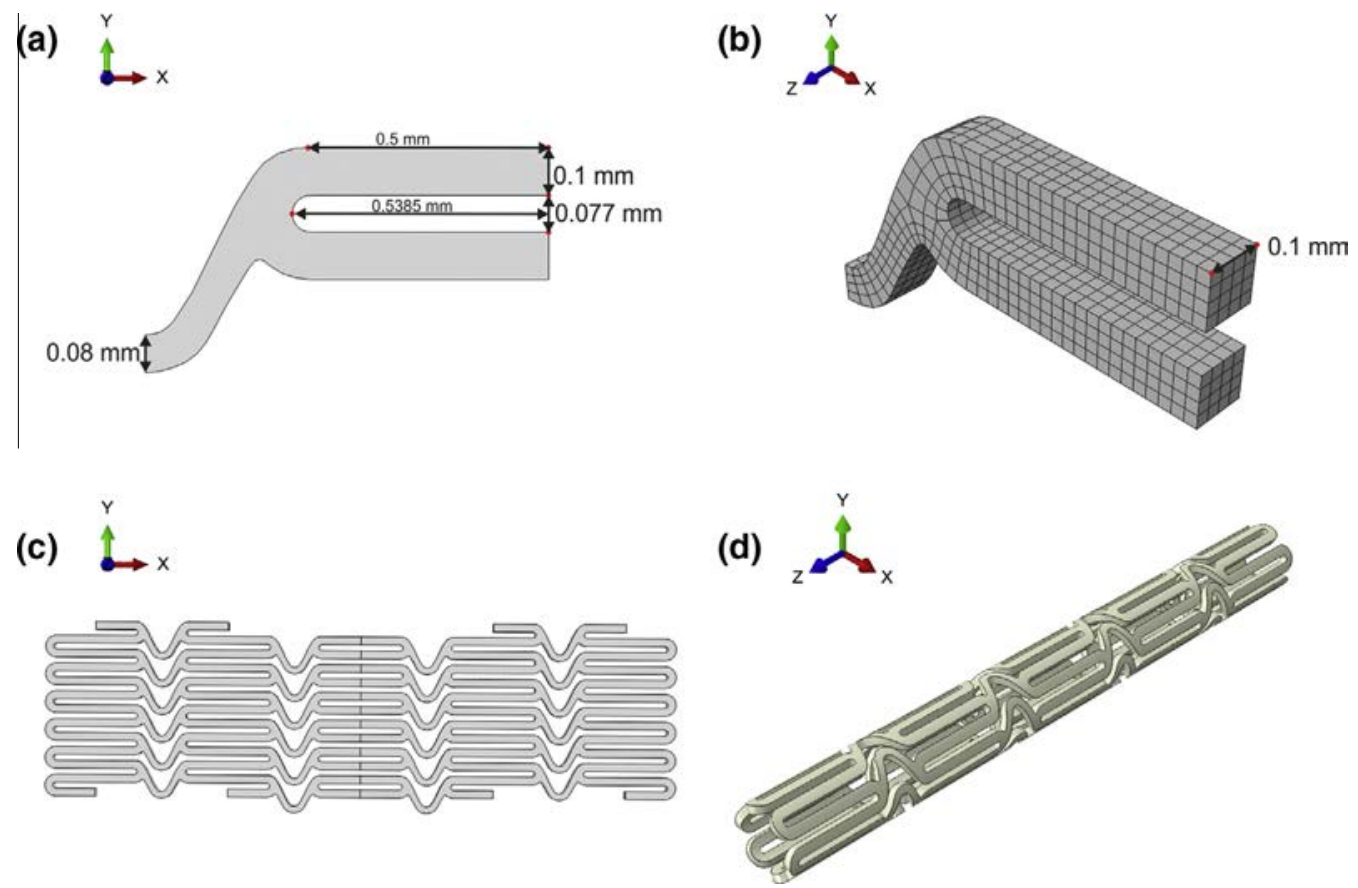

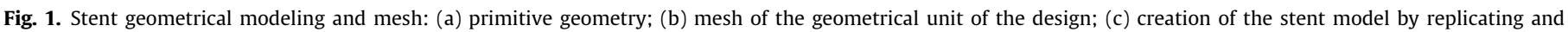
mirroring the mesh of the geometrical unit along the plane; (d) final stent model.

\subsection{Stent: constitutive modeling}

The stent is assumed to be made of a $316 \mathrm{~L}$ stainless steel. Since stent lifetime is affected by the initial deformation due to the crimp and deployment processes and by cyclic loading, the adopted material model has to simulate both monotonic and cyclic behavior of 316L stainless steel [39]. Therefore, we adopt a $J_{2}$ elastoplastic constitutive model with non-linear isotropic and kinematic hardening (with one kinematic center). The constitutive model is already implemented within the material library of the FEA software Abaqus v.6.11 and labeled by the keyword *Plastic, hardening $=$ COMBINED, number backstresses $=1$.

The same model has been used in [47] for the calibration of the fatigue criteria on experiments [27,22,21]; for further details, see [47]. Here we just note that the constitutive model has been calibrated on experimental data related to both monotonic and cyclic behavior of $316 \mathrm{~L}$ stainless steel.

\subsection{Vessel: geometrical modeling, mesh, constitutive modeling}

The coronary artery is modeled as an idealized vessel represented by a thick-walled pipe with an initial inner diameter of $2 \mathrm{~mm}$, wall thickness $0.5 \mathrm{~mm}$ and length $14 \mathrm{~mm}$. The mesh model consists in 19,552 nodes and 14,508 hybrid three-dimensional brick elements (C3D8H) available in the Abaqus v.6.11 element library.

The mechanical response of the artery wall is modeled using a polynomial hyperelastic model, suitable for incompressible isotropic materials and available in the software material library as ${ }^{*}$ Hyperelastic; see [52]. The adopted material parameters stem from the work by Lally et al. [53,54], who performed FEA of coronary stenting to investigate the impact of stent design on vessel tissue solicitation.

\subsection{Analysis set-up}

The device is first mounted onto an angioplasty balloon, through a radial crimping in order to ensure the necessary grip between the device and the delivery system. Subsequently, the bal- loon/stent system is driven to the target vascular region and, once in place, the balloon is inflated forcing the stent deployment. After the acute elastic recoil, the stent acts as a scaffold of the vessel ensuring the lumen patency.

Although comprehensive simulation of stenting would embed all the previously described procedural steps, i.e., crimping, delivery and deployment [55], in the present study we neglect the delivery stage for three specific reasons. From the mechanical point of view, the solicitations experienced by the stent during crimping and deployment are more predominant when compared to those imposed by the stent delivery. Furthermore, given the study aim, our main interest regards the cyclic solicitation experienced by the stent after its implant. Nevertheless, such a choice reduces significantly the computational cost of the analysis.

For such reasons, we split the stenting simulation in two analyses described in the following subsections: the first one accounts for the stent crimping, while the second one addresses the simulation of stent deployment and the subsequent diastolic-systolic cyclic loading within the artery (i.e., fatigue loading).

\subsubsection{Stent crimping}

The FE model of this analysis accounts for two parts: the stent and a rigid cylinder, which is aimed at imposing the reduction of the stent outer diameter; the rigid surface is discretized by 1280 4-node quadrilateral surface elements (SFM3D4) and 1312 nodes.

The analysis is divided in two steps. In the first step the outer diameter of the rigid surface is reduced from $0.9 \mathrm{~mm}$ to $0.72 \mathrm{~mm}$ through a displacement-driven strategy. At the same time, a surface-to-surface contact is activated between the outer surface of the stent and the inner surface of the rigid cylinder. In particular, we adopt a frictionless contact characterized by hard contact pressure-overclosure relationship for the normal component, enforced by linear penalty method (with 0.001 as a scale factor for the default linear penalty stiffness). In the second step, the rigid cylinder is re-enlarged allowing the elastic recoil of the stent, which however keeps partially crimped. At the end of the analysis the outer stent profile is reduced from $0.876 \mathrm{~mm}$ to $0.758 \mathrm{~mm}$. 


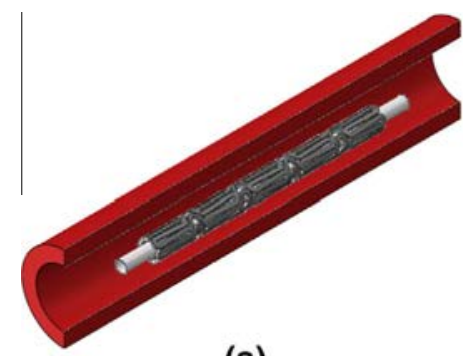

(a)

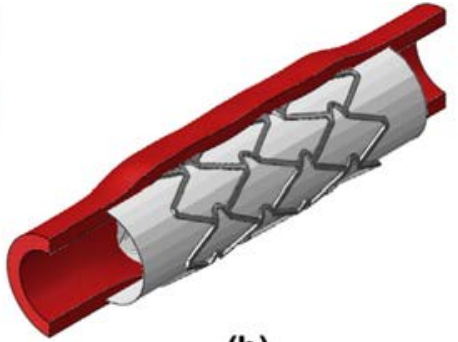

(b)

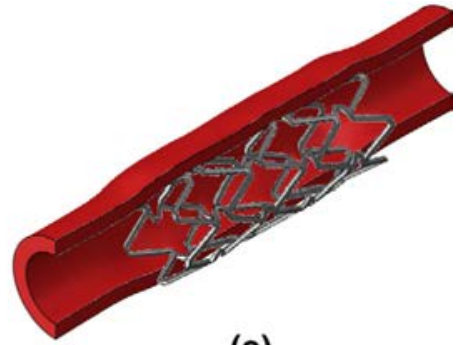

(c)


vessel configuration after balloon deflation.

Appropriate boundary conditions are applied to some stent nodes to avoid rigid motion.

\subsubsection{Stent deployment and diastolic-systolic cyclic loading}

This second stage of the analysis has the final increment of the previous analysis as starting point (see Fig. 2a). In fact, the loaded and deformed configuration of the crimped stent is imported in the simulation environment, which now consists of three model parts: (i) the stent itself, (ii) the vessel, and (iii) the balloon. The vessel and the balloon are modeled as previously discussed (see Section 2.3). The balloon inflation is consequently modeled through a displacement-driven analysis where an uniform radial displacement is imposed to the cylindrical rigid surface imposing the corresponding stent enlargement through the contact with the inner stent surface.

The analysis is divided into the following steps:

1. Vessel inflation: the arterial model is pressurized through the application of an internal pressure of $120 \mathrm{mmHg}$. Assuming a cylindrical coordinate system, axial and circumferential displacements of the vessel ends are fixed, allowing only the radial displacement.

2. Stent expansion: the balloon model is radially enlarged, allowing the stent expansion to the target outer diameter of $3 \mathrm{~mm}$ (see Fig. 2b). In this step two contact pairs are activated: the first between the inner surface of the stent (slave surface) and the outer surface of the balloon model (master surface) and the second between the inner surface of the vessel (slave surface) and the outer surface of the stent (master surface). The contact settings are similar to those adopted for the crimping analysis.

3. Vessel deflation (diastole): in this step the contact between the balloon and the stent is deactivated and the inner pressure reduced to $80 \mathrm{mmHg}$, thus mimicking the diastolic conditions and allowing the stent/vessel system to reach the equilibrium after elastic recoil (see Fig. 2c).

4. Vessel inflation (systole): the internal pressure of artery is again increased to $120 \mathrm{mmHg}$, thus simulating the systolic condition, closing a loading cycle.

The analysis is then completed by ten loading cycles.

\subsubsection{Bending cyclic loading}

In order to investigate the effect of the curved vessel on the stent after deployment, a cyclic bending deformation is also imposed on the expanded stent model and the corresponding bending loading is computed. In this case, the simulation of bending represents the third stage of loading after crimping and free expansion. It is carried according to the procedure described in [56], i.e., the extremes of the stent model are rotated at a fixed angle using a multi-point constraint (MPC) boundary condition
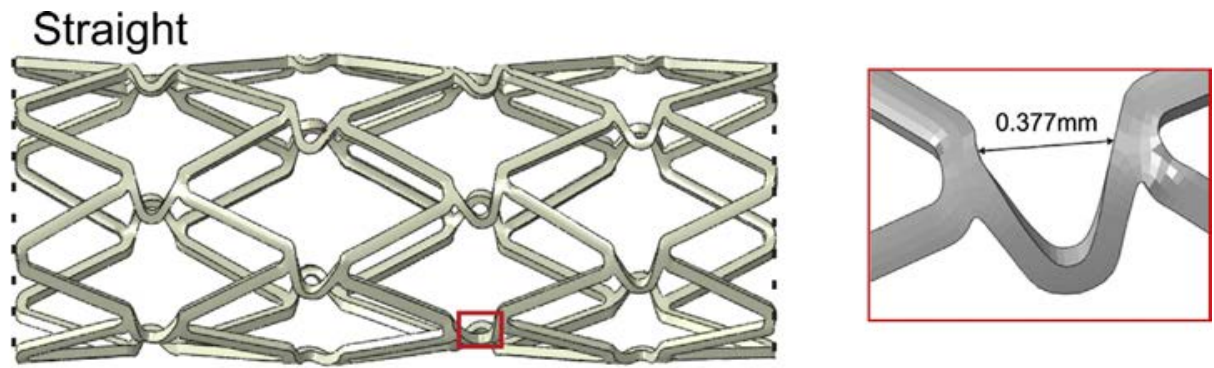

\section{Bent}
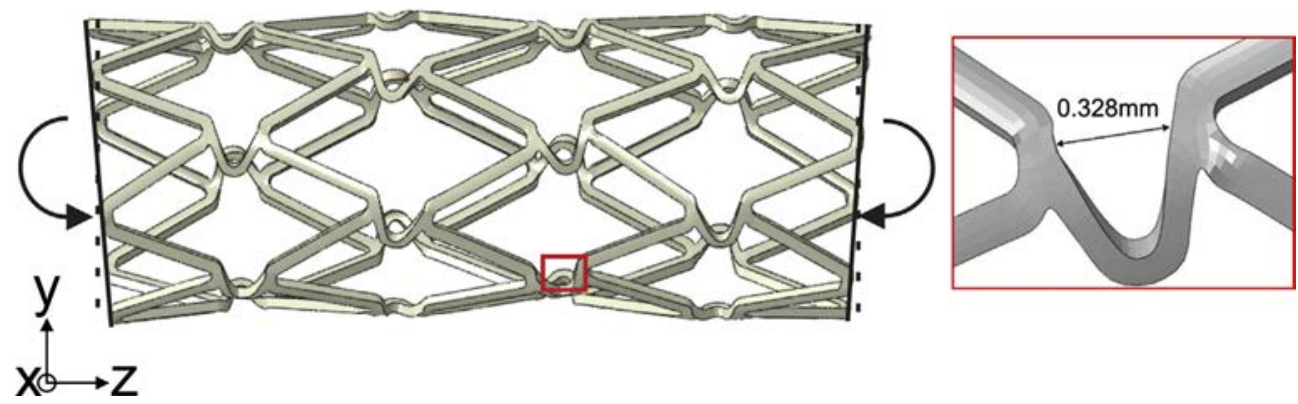



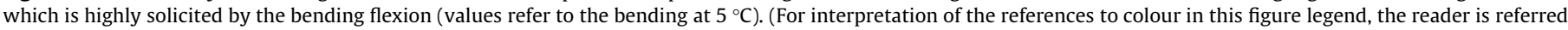
to the web version of this article.) 
Table 1

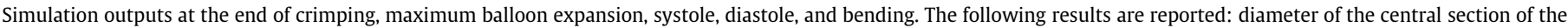


equivalent plastic strains (PEEQ).

\begin{tabular}{|c|c|c|c|c|c|c|c|}
\hline \multirow[t]{2}{*}{ Step } & \multirow[t]{2}{*}{ Diameter [mm] } & \multicolumn{2}{|c|}{ MISES [MPa] } & \multicolumn{2}{|c|}{ PRESS [MPa] } & \multicolumn{2}{|c|}{ PEEQ [\%] } \\
\hline & & $\operatorname{Max}$ & Min & Max & Min & $\operatorname{Max}$ & Min \\
\hline Crimping & 0.737 & 250 & 0.9 & 139 & -126 & 5.5 & 0 \\
\hline Max balloon expansion & 3.022 & 442 & 2.5 & 483 & -476 & 95.8 & 0 \\
\hline Systole & 2.760 & 422 & 3.4 & 253 & -272 & 99.7 & 0 \\
\hline Diastole & 2.758 & 442 & 3.8 & 252 & -272 & 99.7 & 0 \\
\hline Bending $\left(2.5^{\circ} \mathrm{C}\right)$ & 2.870 & 395 & 2.7 & 329 & -292 & 86.0 & 0 \\
\hline Bending $\left(5^{\circ} \mathrm{C}\right)$ & 2.870 & 435 & 3.2 & 444 & -383 & 104.0 & 0 \\
\hline
\end{tabular}

as shown in Fig. 3. Two fixed angles of 2.5 and $5{ }^{\circ} \mathrm{C}$ are considered. The analysis is finally completed by ten loading cycles.

\subsection{Analysis results}

To give an order of magnitude of the main variables values, Table 1 reports several outputs at the end of crimping, maximum balloon expansion, systole, diastole, and bending. As expected, the diameter of the central section of the stent increases from a value of $0.737 \mathrm{~mm}$ during crimping to a value of $3.022 \mathrm{~mm}$ at maximum balloon inflation, while the passage from systole to diastole results in a very low diameter change of $0.002 \mathrm{~mm}$. In the case of bending, the stent outer diameter is $2.870 \mathrm{~mm}$, thus higher than the case of deployment in the straight artery, which radially compresses the device.

The von-Mises equivalent stress (MISES) reaches the maximum value (i.e., $442 \mathrm{MPa}$ ) at maximum balloon inflation; this implies high levels of plastic strain, recalling that the initial yield limit is $211 \mathrm{MPa}$ (see [47]). Similarly, the equivalent pressure stress (PRESS) reaches its (absolute) maximum values at maximum balloon inflation and presents almost constant values during systole and diastole. Moreover, the obtained values at the end of bending (especially the $5{ }^{\circ} \mathrm{C}$ bending case) are very high and comparable to those reached at maximum balloon inflation. In particular, one inter-ring connector is highly solicited by the bending cycling loading, as depicted in the red boxes of Fig. 3. The presented results highlight also very high values of the equivalent plastic strain (PEEQ), especially at maximum balloon inflation and bending, which slightly increase during systole and remain constant during diastole. Such values evidence how both the initial deployment of the stent within the artery and the long-term service loading are important steps which need to be accounted for during stent design and simulation.

In order to further investigate the results presented in Table 1, we focus on the distribution of the variables of interest in a stent strut composed of two axialsymmetric units (the single unit is shown in Fig. 1b).

Residual stresses introduced in the described analysis steps can be clearly seen in the von-Mises equivalent stress distributions along the stent strut of Fig. 4. In particular, we report three views of the stent strut (indicated, respectively, as View 1, View 2, and View 3) at different steps of the FE simulation, i.e., at crimping, maximum balloon expansion, systole, and diastole. High stress gradients are evident at all the steps. After the elastic recoil, the vonMises stress distribution remains mainly at the inner regions of the curved stent strut sections (see View 1 and View 2 for crimping) and

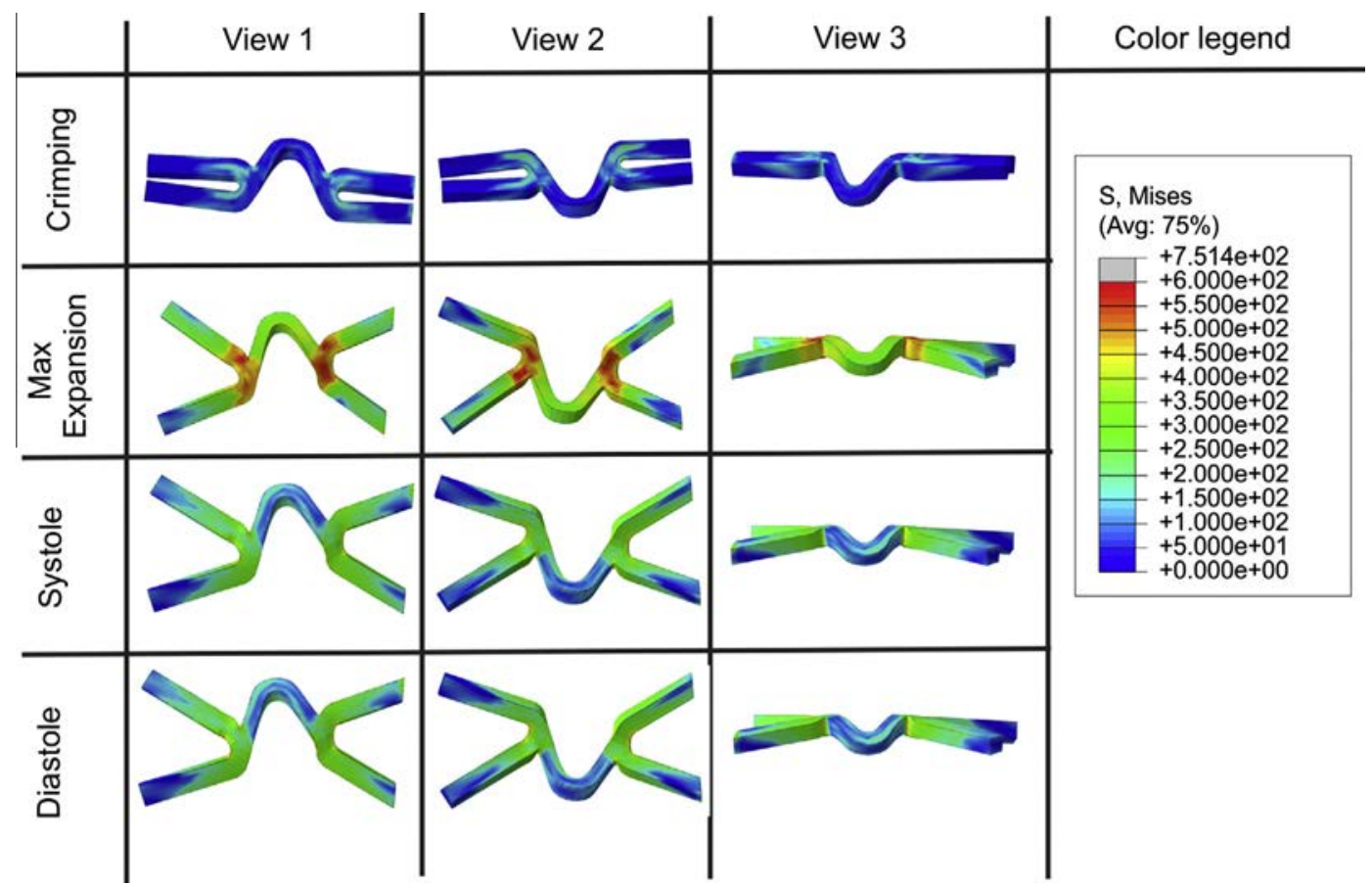

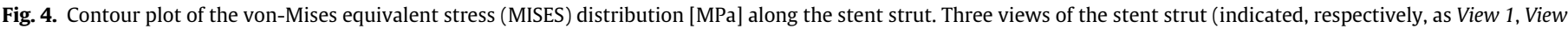
2, and View 3) are reported at different FEA steps, i.e., crimping, maximum balloon expansion, systole, and diastole. 
(a)



Fig. 5. Contour plot of the equivalent pressure stress (PRESS) distribution [MPa] along the stent strut at different FEA steps: (a) at maximum balloon inflation; (b) at diastole peak.

it is particularly concentrated along the thickness of the strut with the lowest curvature radius (see View 3 for crimping). The highest stresses are generated in the same regions at maximum balloon inflation (see View 1, View 2, and View 3 for max expansion). The von-Mises stress distribution during fatigue loading at systole and diastole peaks is almost the same and again shows the highest values at the inner regions of the curved stent strut sections (see View 1, View 2 and View 3 for systole and diastole).

Contour plots of the equivalent pressure stress are presented in Fig. 5a and $\mathrm{b}$ at different analysis steps. The maximum values are again reached at maximum balloon inflation (see Fig. 5a), while decrease at diastole peak (see Fig. 5b). Similarly to the von-Mises stress distribution, the pressure distribution remains mainly at the inner regions of the curved stent strut sections and along the thickness. Such a concentrated distribution of both the von-Mises and pressure stresses will affect the fatigue-life of these regions, as explained in the following Section.

As already mentioned above, high values of the equivalent plastic strain are reached at the maximum balloon expansion (see Table 1). This could imply a failure of material during initial deployment of the stent rather than during fatigue loading. To this purpose, we propose to investigate also the distribution of the equivalent plastic strain along the entire stent during the analysis steps. We remark, moreover, that many works from the literature, dealing with stent fatigue, have mainly focused on variable evolution during fatigue loading without analyzing a possible failure during deployment. Fig. 6 shows the histogram of the equivalent



Fig. 6. Histogram of the equivalent plastic strain (PEEQ) [\%] at different FEA steps, i.e., post crimping, maximum balloon inflation, systole, and diastole. The frequency is defined as the ratio between the number of elements, $N_{e l}$, and the total number of elements of the mesh, $N_{\text {tot }}$. plastic strain at different analysis steps, i.e., at post crimping, maximum balloon inflation, systole, and diastole. The frequency is defined as the ratio between the number of elements, $N_{e l}$, and the total number of elements of the mesh, $N_{\text {tot }}$. The values assumed by the equivalent plastic strain during the analysis are divided into five intervals ranging from $0 \%$ to $100 \%$. As it can be observed, the major percentage of elements (about 90-95\%) presents low equivalent plastic strain values ranging from $0 \%$ to $20 \%$ at all the analysis steps. In particular, $93 \%$ of elements at post crimping, $67 \%$ of elements at maximum balloon inflation, and $65 \%$ of elements at diastole and systole present values between $0 \%$ and $1 \%$. Only few elements $(0.0885 \%$ at max balloon inflation and $0.1163 \%$ at diastole and systole), mainly located in the inner regions of the curved stent strut sections, show values of the equivalent plastic strain in the range $80-100 \%$.

To check the reasonability of our FEA results, we compare the orders of magnitude of the obtained quantities to those provided in $[38,39]$, that investigated the same stent design. In particular, the order of magnitude of the von-Mises stress is similar to that by Sweeney et al. [39], ranging between 0 and $250 \mathrm{MPa}$ after post crimp recoil and between 0 and $700 \mathrm{MPa}$ after post deployment recoil (see Table 1). Additionally, the equivalent pressure stress values reported by McGarry et al. [38] are comparable to our values (see Table 1), although very high peaks (about $4000 \mathrm{MPa}$ ) are highlighted in some localized regions [38]. The accumulated plastic slip by Sweeney et al. $[39,38]$ ranges between $0 \%$ and $100 \%$. Also, the distribution of the reported variables shows the highest values along the curved regions of the stent strut.

Finally, the mechanical FE analysis allows to calculate the variables of interest at the shakedown or stabilized mechanical state reached by the stent under investigation, to be then used for the fatigue analysis (see Section 1). As a result of the FE analysis, we obtain a stabilized cycle under elastic shakedown after diastolicsystolic cyclic loading and under plastic shakedown after cyclic bending. As an example, Fig. 7a shows the equivalent plastic strain versus time diagram for the stent subjected to diastole-systole cyclic loading. Two curves are represented: the blue curve is related to the point where the maximum value of equivalent plastic strain is reached among all the mesh elements; the red curve is related to the point of the analyzed stent strut where the maximum value of equivalent plastic strain is reached. The location of the points is indicated in Fig. 7b. In both cases, the equivalent plastic strain stabilizes on a limit value, thus demonstrating the existence of a stabilized cycle for the stent, i.e., the occurrence of elastic shakedown. This is also clear by recalling the results provided in Table 1 and Fig. 6, which show a constant value of the equivalent plastic strain during diastole and systole. On the contrary, from the bending analysis with an applied angle of $5{ }^{\circ} \mathrm{C}$, part of the elements (about 32\%) result under plastic shakedown. If we apply an angle of $2.5^{\circ} \mathrm{C}$, the number of elements under plastic shakedown reduces to $20 \%$.

\section{Fatigue analysis}

This Section first presents the applied fatigue criteria; then, it presents the results of the fatigue analysis.

\subsection{Fatigue criteria}

Since the stabilized cycle of the stent results under elastic shakedown after diastolic-systolic cyclic loading (see Section 2.5), then we propose to use a Dang Van (DV) based approach [46]. The classical DV criterion allows to define the limit of the imposed external load under which the structure will have an infinite life. To this purpose, Dang Van considered the mesoscopic grain scale 


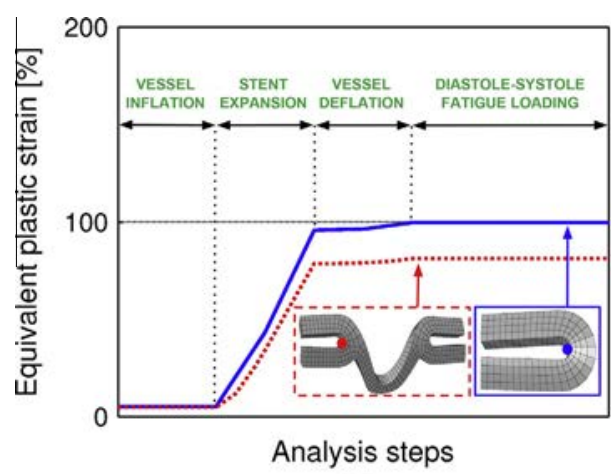

(a)

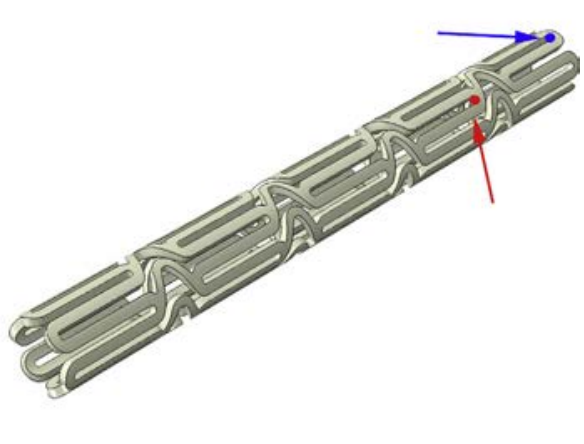

(b)

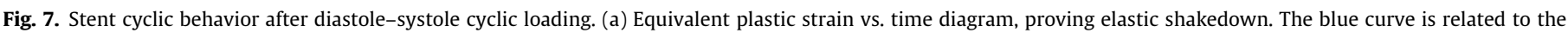



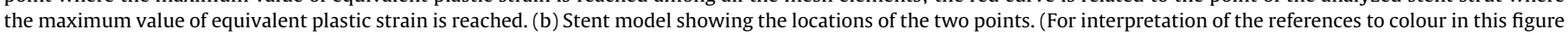
legend, the reader is referred to the web version of this article.)

and the macroscopic structure scale. The relations between mesoscopic and macroscopic fields can be reached, for example, using a homogenization scheme; see, e.g., [57,58]. In particular, by adopting the Lin-Taylor homogenization law, a passage between the macroscopic and the mesoscopic scale [59] allows to state that the lifetime is infinite if the mesoscopic shear stress, $\tau_{\mu}(t)$, and the hydrostatic stress, $\sigma_{h}(t)$, satisfy the following inequality in all the points of the structure (subscript $\mu$ stands for mesoscopic variables):

$\max _{t}\left[\tau_{\mu}(t)+\alpha_{N} \sigma_{h}(t)\right] \leqslant \beta_{N}$

$\alpha_{N}$ and $\beta_{N}$ being material parameters, where subscript $N$ stands for finite lifetime.

The calibration of $\alpha_{N}$ and $\beta_{N}$ for $N=10^{7}$ has been conducted by Auricchio et al. [47] on experimental data related to micrometric specimens, produced with the standard procedure applied in stent manufacturing industry $[22,21,27]$. Such a calibration has been done on the experiments related only to the smooth specimens and not to the notched ones that result in a plastic shakedown state. This means that the investigated stent under diastole-systole loading reveals a conservative behavior, similar to that manifested by the tested smooth specimens.

Here, we propose to analyze the behavior of the stent at the required $4 \cdot 10^{8}$ cycles [13], i.e., we calibrate $\alpha_{N}$ and $\beta_{N}$ for $N=4 \cdot 10^{8}$. We first assume $\alpha_{N}$ equal to that obtained for $N=10^{7}$, as classically done in metal fatigue $[60,61]$. To evaluate $\beta_{N}$, we then extrapolate the fatigue limits at the required cycles from the experimental data provided in [27]. We fit each set of data by Donnelly [27], related to the $50,75,100$, and $150 \mu \mathrm{m}$ width specimens, with a classical Basquin law in the form $S=a \cdot N_{f}^{b}$, where $S$ is the stress amplitude, $N_{f}$ the number of cycles to failure, and $a$ and $b$ material parameters. Table 2 provides the fitted material constants $a$ and $b$. Since both strut thickness and width are approximately $0.10 \mathrm{~mm}$ and the diameter of the stent unit cell is

Table 2

Material parameters $a$ and $b$ of the classical Basquin law in the form $S=a \cdot N_{f}^{b}$, obtained by fitting the experimental curves by Donnelly [27], related to the $50,75,100$, and $150 \mu \mathrm{m}$ width specimens.

\begin{tabular}{lll}
\hline Specimen width $(\mu \mathrm{m})$ & $a(\mathrm{MPa})$ & $b$ \\
\hline 50 & 127.556 & -0.013 \\
75 & 166.285 & -0.016 \\
100 & 159.912 & -0.011 \\
150 & 142.717 & $5 . \mathrm{E}-04$ \\
\hline
\end{tabular}

$0.077 \mathrm{~mm}$ (see Fig. 1a), we will focus on the fitted curve related to the $100 \mu \mathrm{m}$ width specimen. Fig. 8 shows the red line fitting the experimental data for the $100 \mu \mathrm{m}$ width specimen and the related 95\% confidence interval for $a$ and $b$ [62]. As it can be observed, the fatigue limit does not reach an asymptotic value and continues to decrease $[63,64]$. The DV parameter $\beta_{N}$ is then derived; Table 3 lists the DV parameters for all the specimens.

Then, since the stabilized cycle of the stent results under plastic shakedown after bending cyclic loading (see Section 2.5), we adopt the criterion based on the dissipated energy per cycle, defined as follows $[48,49]$ :

$W^{p}=c N_{f}^{b}$

where $W^{p}$ is the dissipated energy per cycle, integrated in each point of the structure over the complete stabilized cycle, as follows:

$W^{p}=\int_{\text {cycle }} \boldsymbol{\sigma}: \dot{\boldsymbol{\varepsilon}}^{p} d t$

Parameter $b$ and $c$ have been calibrated by Auricchio et al. [47] and are equal to -0.4687 and $431.4 \mathrm{MPa}$, respectively.

Both the criteria are computed as post-processing in all the Gauss integration points of the stent model. Particularly, the post-process of the DV criterion is performed with the Matlab implementation of the optimization code SDPT3 [65]. We would like to remark that our fatigue analysis is conservative since we adopt a hot-spot approach in a high gradient zone.

\subsection{Fatigue results}

The mechanical fields have been obtained by the FE simulation described in Section 2.

We start by analyze the results of the diastolic-systolic cyclic loading analysis.

Fig. 9a represents the diagram in terms of the mesoscopic shear stress, $\tau_{\mu}$, and the hydrostatic stress, $\sigma_{h}$, where the calibrated DV line, related to the $100 \mu \mathrm{m}$ width specimen, is represented as red smooth line, while the red dashed lines define the $95 \%$ confidence interval [62]. The upper bound line coincides also with the DV line calibrated by Auricchio et al. [47] for $N=10^{7}$.

From the DV postprocessing, the most critical element is the element $A$ indicated in Fig. 9b. The loading paths due to blood pressure and related to the Gauss integration points of this element are represented by green dashed lines. These loading paths are almost overlapping and only small variations of the mesoscopic shear stress can be noticed. Moreover, all the paths lie under the DV line, 




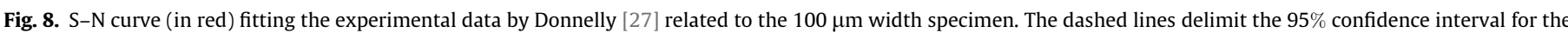
fitting coefficients $a$ and $b$. (For interpretation of the references to colour in this figure legend, the reader is referred to the web version of this article.)

Table 3

Proposed DV parameters, $\alpha_{N}$ and $\beta_{N}$, referring to a fatigue-life $N=4 \cdot 10^{8}$ and calibrated on data related to $50,75,100$, and $150 \mu \mathrm{m}$ width specimens $[21,22,27]$.

\begin{tabular}{llll}
\hline Specimen width $(\mu \mathrm{m})$ & Fatigue-life $N$ & $\alpha_{N}$ & $\beta_{N}(\mathrm{MPa})$ \\
\hline 50 & $4 \cdot 10^{8}$ & 0.4821 & 111.93 \\
75 & $4 \cdot 10^{8}$ & 0.4821 & 137.50 \\
100 & $4 \cdot 10^{8}$ & 0.4821 & 146.84 \\
150 & $4 \cdot 10^{8}$ & 0.4821 & 165.0 \\
\hline
\end{tabular}

thus indicating that the considered stent under diastolic-systolic loading has an endurance limit of $4 \cdot 10^{8}$ cycles. In particular, data from stent FEA reveal high values of the hydrostatic stress, $\sigma_{h}$ and low values of mesoscopic shear, $\tau_{\mu}$. This emphasizes the important role of stress concentrations in stents, due to the existence of small radii and high stress gradients. The von-Mises stress values of Fig. 4 show, in fact, a variation of around $200 \mathrm{MPa}$ along the stent strut at the same instant, while the pressure stress values of Fig. 5 a variation of 200-300 MPa. Moreover, the high values assumed by the pressure stress play a fundamental role in fatigue predictions of stents and need to be accounted for. The elements located in the inner regions of the curved stent strut sections (elements $\mathrm{B}$ and $\mathrm{C}$ of Fig. 9b) are in the safe region of the DV diagram, due to the presence of compressive residual stresses $(\sim 200 \mathrm{MPa})$.

In order to highlight the most solicited parts of the stent strut, we compute the following DV fatigue damage parameter:

$C_{D V}=\max _{t} \frac{\tau_{\mu}(t)+\alpha_{N} \sigma_{h}(t)-\beta_{N}}{\beta_{N}}$

Positive values of the DV parameter, $C_{D V}$, indicate failure, while negative values indicate no failure. Fig. 10 shows the isovalues of the DV parameter, $C_{D V}$, calibrated with respect to the $100 \mu \mathrm{m}$ DV line, along the stent strut. As it can be observed, the distribution of $C_{D V}$ is almost uniform in the stent strut. The critical points (see the zoom at the bottom of Fig. 10) are located along the thickness in the region with the lowest curvature radius (see element $A$ of Fig. 9b), where a value of -0.14 is reached. It is worth to highlight that the conducted three-dimensional analysis allows to take into account variable distributions and gradients along the stent thickness and thus to localize possible critical points along the stent thickness for the fatigue-life assessment.

Then, we focus on the results of the bending analysis.

The fatigue analysis is here driven by the elements under plastic shakedown, located in the connector regions of the stent. In particular, the most critical element is located in the highly solicited



(a)

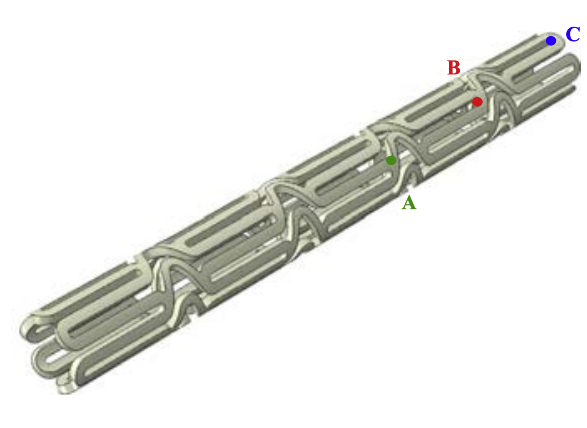

(b)



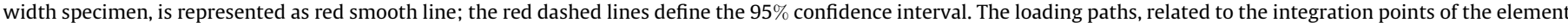

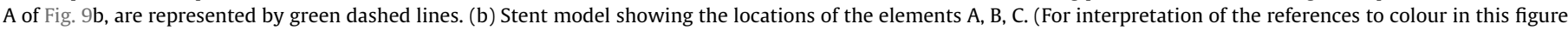
legend, the reader is referred to the web version of this article.) 

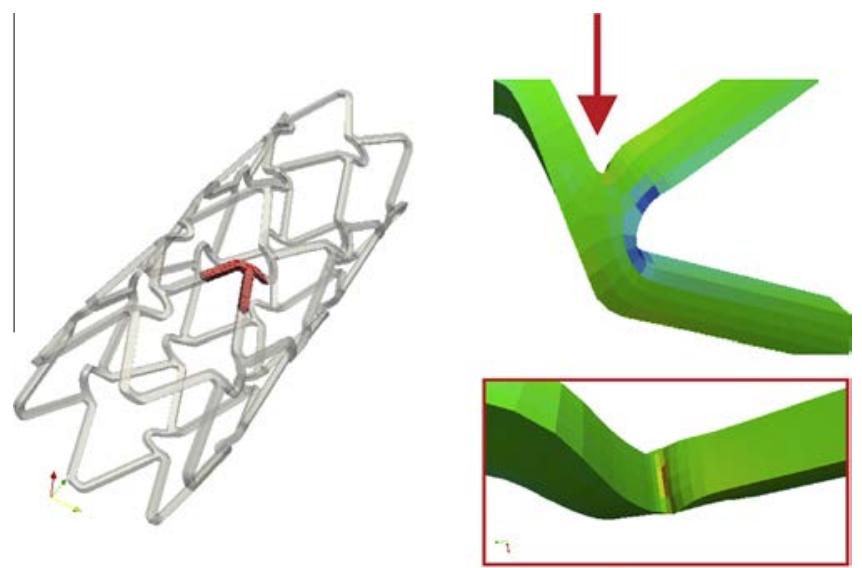

$-1.8-1.6$

$-1.2$

$-0.8$

$-0.4$ 0

Fig. 10. Application of the Dang Van (DV) criterion to the stent strut (at the top). Isovalues of the DV parameter, $C_{D V}$, computed with respect to the DV line calibrated on $100 \mu \mathrm{m}$ width specimens (negative values indicate no failure; positive values indicate failure). Zoom of the region (at the bottom) where the critical points are located (element A of Fig. 9b).

inter-ring connector depicted in the red boxes of Fig. 3. In this region, plasticity is very localized and high gradients are observed. The fatigue life associated to this element is equal to 6590 and 343 cycles for an applied angle of 2.5 and $5{ }^{\circ} \mathrm{C}$, respectively. As already pointed out, our approach to fatigue is conservative since based on a hot-spot evaluation of fatigue variables; if we assume a $50 \%$ reduction of the dissipated energy (see [47]), the fatigue life is equal to 28,898 and 1500 cycles for an applied angle of 2.5 and $5{ }^{\circ} \mathrm{C}$, respectively. However, at present we have no information about stent lifetime under such a loading condition; we recall in fact that the requested $4 \cdot 10^{8}$ cycles refers to systolic-diastolic pulsatile loading. Therefore, not enough data are available to interpret the estimated lifetime. Further investigations about the effect of such a type of loading will be needed, especially in terms of comparison with purposely-designed experimental data. Moreover, we need to highlight that in the bending analysis we have considered a cyclic loading by imposing two purely arbitrary angles on the stent and by neglecting the real interaction with a soft material vessel.

Further, in order to give an idea of the loading paths in the DV diagram for the elements under elastic shakedown, Fig. 11a represents the paths (dashed green lines) related to two Gauss integration points of the element $\mathrm{A}$ indicated in Fig. 9b, which is the



Fig. 11. Application of the Dang Van (DV) criterion to the stent under bending loading in the $\left(\sigma_{h}, \tau_{\mu}\right)$ plane. The proposed DV line (in red), related to the $100 \mu \mathrm{m}$ width specimen, is represented as red smooth line; the red dashed lines define the 95\% confidence interval. The loading paths, related to two integration points of element A of Fig. 9b, are represented by green dashed lines. (For interpretation of the references to colour in this figure legend, the reader is referred to the web version of this article.) critical element for the blood pressure loading case. On the one hand, we can observe a complete qualitative and quantitative change of the stress path between pulsatile pressure and bending. On the other hand, we can remark that the variability of the paths corresponding to two neighboring Gauss points illustrates the importance of the stress gradient in such a region.

\section{Conclusions and perspectives}

This paper has proposed a numerical design method for the lifetime prediction of stents. The methodology has been illustrated through the analysis of an idealized generic balloon-expandable stent; as such, the analysis should be understood as a general discussion and not as a specific guideline on stent geometry or clinical scenarios.

The effects of cycling loading including blood pressure and bending due to vessel curvature have been explored; the most critical elements have been identified in hinge points and inter-ring connectors for pulsatile and bending loading, respectively. The effect of bending cyclic loading has been quantified more dangerous than the effect of the blood pressure. The effect of the pulsatile pressure has shown in fact a percentage diameter change lower than $0.08 \%$ that corresponds to very small amplitudes for the cyclic stresses (see Table 1); these amplitudes correspond to quasistatic loading conditions for the stent.

The obtained results provide however useful information either to be used for product improvement or for clinicians to make lifesaving decisions and encourage further investigation of such an approach for future research developments in the framework of the numerical fatigue-life assessment of stents. This approach is in fact general and not limited to 316L stainless steel: further applications could concern other metallic materials as cobaltchromium alloys. The methodology should be also considered as an incentive to explore other loading scenarios.

The proposed methodology is however based on simplifications that are listed in the following and that can be accepted since the main aim of the study is the analysis of the mechanical and fatigue behavior of a generic stent design.

Firstly, the present approach is based on a purely macroscopic continuum material model for the 316L stainless steel, which has been largely applied to stents by continuum mechanics based methodologies [36,37]. Such an approach does not explicitly capture microstructural effects on grain plastic deformation and lifetime $[39,43,66]$; here, we simply assume that the size effect is taken into account through the fatigue-life estimations based on $\mu \mathrm{m}$-size experiments [47]. The idea of introducing the material variability as a probability density function will be the object of future work.

Secondly, as the first main goal of the study is to propose a fatigue-life numerical method for the analysis of balloon-expandable stents, and not an accurate simulation of the stent implant, the adopted modeling procedure does not take into account the actual in vivo behavior of the stent. The material modeling of the arterial tissue neglects arterial stratifications and anisotropy [55]; similarly, the modeling of the plaque is neglected. We recall that current standard guidelines for non-clinical engineering tests [13] do not require vessel and plaque inclusion in the performance analysis. To adopt such methodology in the clinical field, we should include patient-specific image-based geometries [8]. Also, although the inclusion of the balloon can provide a more realistic representation of the transient deformation during the deployment $[31,67]$, the balloon is herein modeled as an expanding cylinder. As for the loading conditions, systolic-diastolic pulsatile pressure and arterial bending are not the only clinical force/deformation modes that must be addressed in bench testing or analysis. Other cyclic defor- 
mation modes, e.g., crushing, axial tension/compression, and torsion, can occur at various frequencies and phases, appearing due to the different physiological forcing functions (e.g., cardiac, respiratory, locomotion), as well as the physiological environment, in particular humidity and corrosion conditions, should also be accounted for. Further developments of the present work will focus on the impact evaluation of these aspects on the computation of the stent stress state and the related fatigue-life assessment.

Thirdly, the adopted multiaxial HCF criterion by Dang Van, based on a micro-macro approach, meets the need of microstructural representation in crack initiation predictions $[68,69]$. Obviously, inhomogeneity effects observed in random crystalline microstructure modeling cannot be captured by the DV approach. However, the size effect is taken into account through the fatigue-life estimations based on $\mu \mathrm{m}$-size experiments.

Finally, to properly validate the proposed computational approach, we should accurately compare the presented numerical predictions with the results of purposely-designed experimental tests.

\section{Acknowledgements}

This work is partially funded by: the Cariplo Foundation through the Project No. 2009.2822; ERC Starting Grant through the Project ISOBIO: Isogeometric Methods for Biomechanics (No. 259229); Ministero dell'Istruzione, dell'Università e della Ricerca through the Project No. 2010BFXRHS; the Italian-French University (UIF-UIF) through the 'Bando Galileo 2013-2014' Grant No. 14830174TJ.

Moreover, Regione Lombardia and CINECA Consortium through a LISA Initiative (Laboratory for Interdisciplinary Advanced Simulation) 2013 grant are gratefully acknowledged.

\section{References}

[1] AL-Mangour B, Mongrain R, Yue S. Coronary stents fracture: an engineering approach (review). Mater Sci Appl 2013;4:606-21.

2] AHA, Circulation 121, e46-e215 2010.

[3] Disegi J, Kennedy R, Pilliar R. Cobalt-base alloys for biomedical applications. ASMT International; 1999.

[4] Petrini L, Migliavacca F. Biomedical applications of shape memory alloys. J. Metall. 2011:2011:1-15.

[5] Winters G, Nutt M. Stainless steel for medical and surgical applications. ASMT International; 2003.

[6] Bombac D, Brojan M, Fajfar P, Kosel F, Turk R. Review of materials in medical applications. Mater Geoenv 2007:54:471-99.

[7] Moravej M, Mantovani D. Biodegradable metals for cardiovascular stent application: interests and new opportunities. Int J Molecul Sci 2011:12(7):4250-70.

[8] Morlacchi S, Pennati G, Petrini L, Dubini G, Migliavacca F. Influence of plaque calcifications on coronary stent fracture: a numerical fatigue life analysis including cardiac wall movement. J Biomechan 2014;47:899-907.

[9] Hibbert B, OBrien ER. Coronary stent fracture. CMAJ: Can Med Assoc J 2011;183(11):E756. http://dx.doi.org/10.1503/cmaj.101078.

[10] Shaikh F, Maddikunta R, Djelmami-Hani M, Solis J, Allaqaband S, Bajwa T. Stent fracture, an incidental finding or a significant marker of clinical in-stent restenosis? Catheter Cardiovasc Interv 2008;71(5):614-8.

[11] Sianos G, Hofma S, Ligthart J, Saia F, Hoye A, Lemos P, Serruys P. Stent fracture and restenosis in the drug-eluting stent era. Catheter Cardiovasc Interv 2004:61(1):111-6.

[12] Nakazawa G, Finn AV, Vorpahl M, Ladich E, Kutys R, Balazs I, Kolodgie FD, Virmani R. Incidence and predictors of drug-eluting stent fracture in human coronary artery: a pathologic analysis. J Am College Cardiol 2009;54(21):1924-31.

[13] US Food and Drug Administration, Non-clinical engineering tests and recommended labeling for intravascular stents and associated delivery systems: guidance for industry and FDA staff, US Department of Health and Human Services; Food and Drug Administration, Centre for Devices and Radiological, Health (April 18th 2010).

[14] Schijve J. Fatigue of structures and materials. Netherlands: Springer Science+Business Media, B.V.; 2009.

[15] Dumoulin C, Cochelin B. Mechanical behaviour of balloon expandable stents. J Biomech 2000;33:1461-70.
[16] Aziz S, Morris J, Perry R, Stables R. Stent expansion: a combination of delivery balloon underexpansion and acute stent recoil reduces predicted stent diameter irrespective of reference vessel size. Heart 2007;93:1562-6.

[17] Harewood F, McHugh P. Modeling of size dependent failure in cardiovascular stent struts under tension and bending. Annal Biomed Eng 2007;35(9):1539-53.

[18] Murphy B, Cuddy H, Harewood F, Connolley T, McHugh P. The influence of grain size on the ductility of micro-scale stainless steel stent struts. J Mater Sci: Mater Med 2006:17(1):1-6.

[19] You X, Connolley T, Mchugh P, Cuddy H, Motz C. A combined experimental and computational study of deformation in grains of biomedical grade 316LVM stainless steel. Acta Materialia 2006;54:4825-40.

[20] Connolley T, McHugh PE, Bruzzi M. A review of deformation and fatigue of metals at small size scales. Fatigue Fract Eng Mater Struct 2005;28(12):1119-52.

[21] Wiersma S, Dolan F, Taylor D. Fatigue and fracture in materials used for microscale biomedical components. Bio-med Mater Eng 2006;16(2):137-46.

[22] Wiersma S, Taylor D. Fatigue of materials used in microscopic components. Fatigue Fract Eng Mater Struct 2005;28(12):1153-60.

[23] Weiss S, Meissner A. Fatigue and microstructure of coronary artery stents. Materialwissenschaft und Werkstofftechnik 2006;37(9):755-61.

[24] Weiss S, Meissner A, Fischer A. Microstructural changes within similar coronary stents produced from two different austenitic steels. J Mech Behav Biomed 2009;2:210-6.

[25] Weiss S, Szymczak H, Meissner A. Fatigue and endurance of coronary stents Materialwissenschaft und Werkstofftechnik 2009;40(1-2):61-4.

[26] Glenn R, Lee J. Accelerated pulsite fatigue testing of Ni-Ti coronary stents. In: Pelton A, Hodgson D, Russell S, Duerig T, editors. Proceedings of the second international conference on shape memory and superelastic technologies (SMST 1997). Pacific Grove, California, CA (USA): SMST Society; 1997.

[27] Donnelly E. Geometry effect in the fatigue behaviour of microscale 316L stainless steel specimens, Ph.D. thesis, National University of Ireland, Galway; 2012.

[28] Cao J, Zhuang W, Wang S, Ho K, Zhang N, Lin J, et al. An integrated crysta plasticity FE system for microforming simulation. J Multiscale Mode 2009;1:107.

[29] Wang S, Zhuang W, Balint D, Lin J. A crystal plasticity study of the necking of micro-films under tension. J Multiscale Modell 2009;1:331.

[30] Auricchio F, Di Loreto M, Sacco E. Finite-element analysis of a stenotic artery revascularization through a stent insertion. Comput Methods Biomechan Biomed Eng 2001;4(3):249-63.

[31] De Beule M, Mortier P, Carlier S, Verhegghe B, Van Impe R, Verdonck P. Realistic finite element-based stent design: the impact of balloon folding. Biomech 2008:41(2):383-9.

[32] Ju F, Xia Z, Sasaki K. On the finite element modelling of balloon-expandable stents. J Mech Behav Biomed Mater 2008;1(1):86-95.

[33] Martin D, Boyle F. Finite element analysis of balloon-expandable coronary stent deployment: influence of angioplasty balloon configuration. Int J Numer Method Biomed Eng 2013; x. XX-xx.

[34] Grogan J, Leen S, McHugh P. Influence of statistical size effects on the plastic deformation of coronary stents. J Mech Behav Biomed 2013;20:61-76.

[35] Li J, Luo Q, Xie Z, Li Y, Zeng Y. Fatigue life analysis and experimental verification of coronary stent. Heart Vessels 2010;25(4):333-7.

36] dos Santos H, Auricchio F, Conti M. Fatigue life assessment of cardiovascula balloon-expandable stents: a two-scale plasticity-damage model approach. J Mech Behav Biomed 2012;15:78-92.

[37] Barrera O, Makradi A, Abbadi M, Azaouzi M, Belouettar S. On high-cycle fatigue of 316L stents. Comput Methods Biomech Biomed Eng 2012:0:1-12.

[38] McGarry J, Ø'Donnell B, McHugh P, McGarry J. Analysis of the mechanica performance of a cardiovascular stent design based on micromechanical modelling. Computat Mater Sci 2004;31:421-38.

[39] Sweeney C, McHugh P, McGarry J, Leen S. Micromechanical methodology for fatigue in cardiovascular stents. Int J Fatigue 2012;44:202-16.

[40] Azaouzi M, Makradi A, Belouettar S. Fatigue life prediction of cardiovascular stent using finite element method. Comput Methods Biomech Biomed Eng 2012;15(S1):93-5.

[41] Schievano S, Taylor A, Capelli C, Lurz P, Nordmeyer J, Migliavacca F, Bonhoeffer P. Patient specific finite element analysis results in more accurate prediction of stent fractures: application to percutaneous pulmonary valve implantation. J Biomech 2010;43(4):687-93.

[42] Lemaitre J, Chaboche J. Mechanics of solid materials. Cambridge University Press; 1994.

[43] Sweeney C, O’Brien B, McHugh P, Leen S. Experimental characterisation for micromechanical modelling of $\mathrm{CoCr}$ stent fatigue. Biomaterials 2014;35:36-48.

[44] Marrey R, Burgermeister R, Grishaber R, Ritchie R. Fatigue and life prediction for cobalt-chromium stents: a fracture mechanics analysis. Biomaterials 2006;27:1988-2000.

[45] Lemaitre J, Sermage J, Desmorat R. A two scale damage concept applied to fatigue. Int J Fract 1999;97:67-81.

[46] Dang Van K. High-cycle metal fatigue in the context of mechanical design. In: Van K, Papadopoulos I. editors, CISM Courses and Lectures no 392. SpringerVerlag, 1999, pp. 57-88.

[47] Auricchio F, Constantinescu A, Scalet G. Fatigue of 316L stainless steel notched um-size components. Int J Fatigue 2014;68:231-47. 
[48] Korsunsky A, Dini D, Dunne F, Walsh M. Comparative assessment of dissipated energy and other fatigue criteria. Int J Fatigue 2007;29(9-11):1990-5.

[49] Charkaluk E, Constantinescu A. Dissipative aspects in high cycle fatigue. Mech Mater 2009;41(5):483-94.

[50] Shechter G, Resar J, McVeigh E. Displacement and velocity of the coronary arteries: cardiac and respiratory motion. IEEE Trans Med Imag 2006;25(3):369-75.

[51] Aoki J, Nakazawa G, Tanabe K, Hoye A, Yamamoto H, Nakayama T, Onuma Y, Higashikuni Y, Otsuki S, Yagishita A, Yachi S, Nakajima H, Hara K. Incidence and clinical impact of coronary stent fracture after sirolimus-eluting stent implantation. Catheterizat Cardiovascular Intervent 2007;69:380-6.

[52] SIMULIA, Abaqus 6.12. Analysis User’s Manual 2013.

[53] Lally C, Dolan F, Prendergast P. Cardiovascular stent design and vessel stresses: a finite element analysis. J Biomech 2005;38(8):1574-81.

[54] Lally C, Dolan F, Prendergast P. Cardiovascular stent design and vessel stresses: a finite element analysis. J Biomech 2006;39:1760.

[55] Mortier P, Holzapfel G, Beule M, Loo D, Taeymans Y, Segers P, Verdonck P, Verhegghe B. A novel simulation strategy for stent insertion and deployment in curved coronary bifurcations: comparison of three drug-eluting stents. Ann Biomed Eng 2010;38(1):88-99.

[56] Petrini L, Migliavacca F, Auricchio F, Dubini G. Numerical investigation of the intravascular coronary stent flexibility. J Biomechan 2004;37:495-501.

[57] Cano F, Constantinescu A, Maitournam H. Critere de fatigue polycyclique pour des materiaux anisotropes: application aux monocristaux. C R Mecanique 2004;332(2):115-21.

[58] Monchiet V, Charkaluk E, Kondo D. A plasticity-damage base micromechanical modelling in high cycle fatigue. C $R$ Mecanique 2006;334(2):129-36.
[59] Dang Van K, Griveau B, Message O. On a new multiaxial fatigue limit criterion: theory and application. In: Brown M, Miller K. editors, Biaxial and Multiaxial Fatigue (EGF 3). London: Mechanical Engineering Publications; 1989, pp. 479496.

[60] Ferjani M, Averbuch D, Constantinescu A. Semianalytical solution for the stress distribution in notched tubes. Int J Fatigue 2011;33(4):557-67.

[61] Ferjani M, Averbuch D, Constantinescu A. A computational approach for the fatigue design of threaded connections. Int J Fatigue 2011;33:610-23.

[62] Kendal M, Stuart A. The advanced theory of statistics, Vol. 1. Hafner Publishing Company; 1963.

[63] Bathias C, Drouillac L, Franois PL. How and why the fatigue s-n curve does not approach a horizontal asymptote. Int J Fatigue 2001;23(Supplement 1):143-51.

[64] Marines I, Bin X, Bathias C. An understanding of very high cycle fatigue of metals. Int J Fatigue 2003;25(9-11):1101-7.

[65] Tütüncü RH, Toh KC, Todd MJ. Solving semidefinite-quadratic-linear programs using SDPT3. Math Program 2003;95(2):189-217.

[66] Sweeney C, Vorster W, Leen S, Sakurada E, McHugh P, Dunne F. The role of elastic anisotropy, length scale and crystallographic slip in fatigue crack nucleation. J Mech Phys Solids 2013;61:1224-40.

[67] Gervaso F, Capelli C, Petrini L, Lattanzio S, Di Virgilio L, Migliavacca F. On the effects of different strategies in modelling balloon-expandable stenting by means of finite element method. J Biomech 2008;41:1206-12.

[68] Guerchais R, Robert C, Morel F, Saintier N. Micromechanical study of the loading path effect in high cycle fatigue. Int J Fatigue 2014;59:64-75.

[69] Bertolino G, Constantinescu A, Ferjani M, Treiber P. A multiscale approach of fatigue and shakedown for notched structures. Theor Appl Fract Mec 2007;48(2):140-51. 